\title{
A Modified Video Codec for MBMS Applications
}

\author{
Praveenkumar Sanigepalli ${ }^{\dagger}$, Hari Kalva ${ }^{\ddagger}$, and Borko Furht ${ }^{\ddagger}$ \\ ${ }^{\dagger}$ Motorola Inc, Plantation, FL, USA, ${ }^{\ddagger}$ Florida Atlantic University, Boca Raton, FL, USA
}

\begin{abstract}
Multimedia Broadcast Multicast Service (MBMS) is currently being standardized by the 3GPP. The $M B M S$ services will enable new class of applications with efficient use of spectrum. MBMS also poses a new set of requirements on video coding and delivery. In this paper we present a short overview of MBMS and a video codec designed for MBMS. The proposed video codec addresses the two key issues in video delivery over MBMS: 1) error resilience and 2) resource adaptive playback. We show that the proposed codec is simple to implement on low-resource terminals such as mobile phones and outperforms MPEG-4 SP coding under lossy conditions.
\end{abstract}

\section{INTRODUCTION}

$\mathrm{C}$ URRENT research on Video Compression algorithms focus on optimizing the compression efficiency [1]. Research in the area of error resilience of compressed video streams target errors over the air or network [2] and do not take into account the resource constraints of devices. A resource constrained mobile device may have to drop video frames prior to decoding, thus resulting in error propagation due to the use of incorrect reference frames. It is essential to devise video compression algorithms that not only support error resilience but also enable video adaptation at the receiver for constraints such as CPU capacity and available power. The ongoing MBMS standardization activity is specifying tools and technologies for multimedia services over mobile networks. The MBMS architecture places a new set of requirements on video codecs to be able to serve a divers set of receivers with varying link conditions and device capabilities [12]. In this paper, we propose a video codec that addresses the two key issues in video delivery over MBMS: 1) error resilience and 2) resource adaptive playback. The proposed video codec reduces the prediction dependencies present in traditional MPEG coding to enable error resilience and receiver side. We evaluate the performance of the codec for different frame loss rates. We show that the proposed method performs significantly better compared to the traditional MPEG coding.
All the MPEG and H.26x video coding standards use hybrid video coding that exploits spatial, temporal and statistical redundancies to achieve high compression rates. The predictive coding techniques used in MPEG video create frame dependencies that result in propagation of any errors that are a direct result of losses in the network or frame dropping at the receiver. H.263+ specifies a reference picture selection (RPS) mode where a past frame is referenced at the encoder for prediction if decoder reference frame is distorted [2]. However, such a technique requires feedback from the decoder channel that is not available in broadcast or multicast applications.

H.263+ has Video Redundancy Coding (VRC) as one of error-resilient encoding mechanisms to suppress the temporal error propagation. The algorithm is to send at least two threads of P-frames simultaneously where each of these P-frames depend on the earlier P-frame of the thread but not on any information of the other thread(s) [4]. Since each thread has to be decoded in a sequence and does not allow smoother resource adaptation. Any packet loss is propagated until the sync frame.

While H.264 supports improved error resilience through multi-frame prediction [5], it is significantly more complex compared to MPEG-4 and resource considerations would require MPEG-4 or H.263 on certain mobile phones. H.264 standard specifies prediction from multiple previous frames for efficiency reasons and the error resilience aspect of such scheme is an actively researched topic.

MPEG-4 supports several tools for error resilience such as RVLC, Intra Block refresh and Data Partitioning [8][9]. These tools effective for combating networks errors but are not effective for resource adaptation.

The rest of the paper is organized as follows: section II gives a brief overview of MBMS, section III presents the proposed video codec. The experimental results and discussion is presented in section IV and conclusions in section V.

\section{OVERVIEW OF MBMS}

3GPP is currently working on a standard for MBMS. This would standardize several components and interfaces in the system architecture that would eliminate ambiguity and promote synergy between network operators, content 
providers, handset manufacturers and network manufacturers [13][14].

Streaming of live or stored video content to group of mobile devices comes under the scope of MBMS. Some of the typical applications are subscription to televised games, music videos, news clips, weather information and live TV content. MBMS specifies transmission of data packets from single entity to multiple recipients using a common broadcast channel. This is much more efficient than IP multicast where packets are duplicated for each recipient in a broadcast/ multicast group. The broadcast service is typically free within the broadcast area whereas multicast service requires subscription. Several multicast groups could exist within the broadcast area where multiple sessions could be ongoing at the same time.

Figure 1 shows an example of a multicast broadcast network. This shows the functionalities required for broadcasting such as QoS handling and where they are implemented within the core network. This architecture enables services offered on the internet or offered by the operator to be broadcast to the terminals.

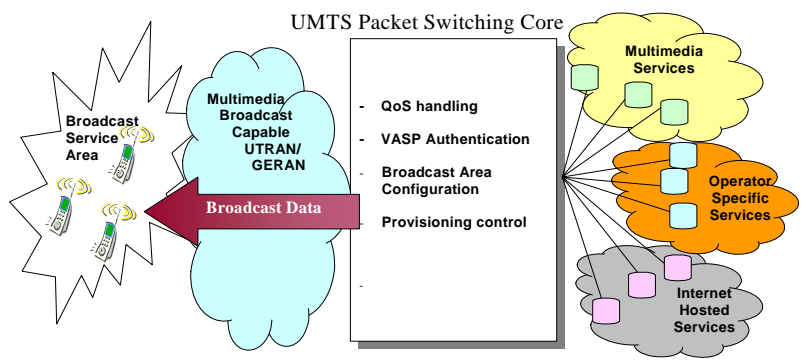

Figure 1: Example of Multicast Broadcast Mode Network

Figure 2 shows the architecture of GSM / UMTS network that would enable such MBMS ser-vices. The Broadcast Multicast Service Controller (BMSC) provides functions for MBMS user service provisioning and delivery to the content provider. It can also serve as an entry point for IP MBMS data traffic from the MBMS User Service source. The GGSN serves as an entry point for IP multicast traffic as MBMS data from the BM-SC. Some of the proposed video error resilience techniques for broadcasting in this paper could be applied either at UMTS Radio Access Network (UTRAN) or GSM/EDGE Radio Access Network (GERAN) or at the server providing service and are depicted in Figure 2.

Figure 2 depicts a Video Resilience Adaptation function such as increasing intra block rates etc can be applied either at Multicast Broadcast Source (MBS) or either at UTRAN or GERAN. By applying at MBS, it would be standard compliant however the adaptation has to take into account the entire multicast group or groups.

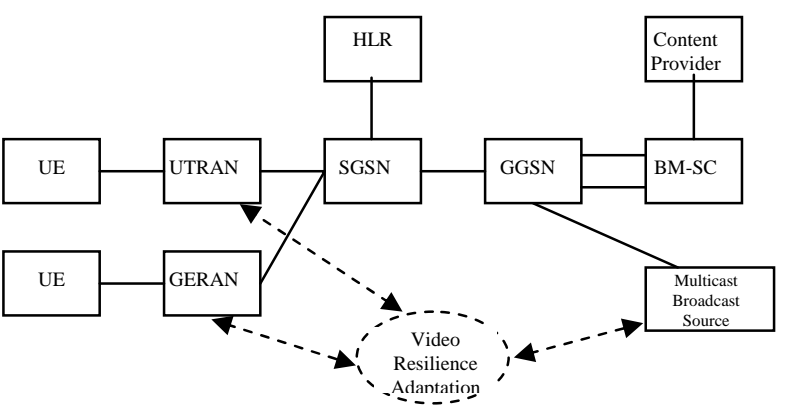

Figure 2: Architecture of MBMS broadcasting over GSM / UMTS network

A large group tends to have heterogeneous link characteristics and any adaptive schemes becomes quite complex or might not improve performance as the source. A partitioning of the group into multiple groups based on loss characteristics at the source is another possibility however this requires more bandwidth on the infrastructure backbone of wire-line network.

Even though adaptation at UTRAN / GERAN serves better as the group size served within the cell will be smaller (for multicast scenarios, it would in the order of tens of users per channel) but this would require provisioning of adaptation at all GERAN/UTRAN. This would additional cost to the infrastructure provider and is not part of the standard. It also requires the MBS to broadcast high quality video to UTRAN/GERANs. For example, video compressed with all I frames at high quality rate such as 30fps can be sent which UTRA/GERAN can adapt based on their individual multicast group characteristics. This requires more bandwidth on wireline net-work and computational complexity/cost at the UTRAN/GERANs. However, the adaptation is more effective as loss rates are homogeneous and response time between mobiles and infrastructure is also faster.

\section{A. MBMS Protocols and Codecs}

In this subsection, we give an overview of the MBMS standard focusing on the streaming aspects as they are applicable to multicasting. However, MBMS also standardizes the download and play services aspects that are not addressed in this paper. Figure 3 shows the components involved for MBMS streaming services where we omitted the security related aspects for simplicity. We give an overview of the MBMS protocols, Codecs, FEC model and quality metrics in four subsections. 


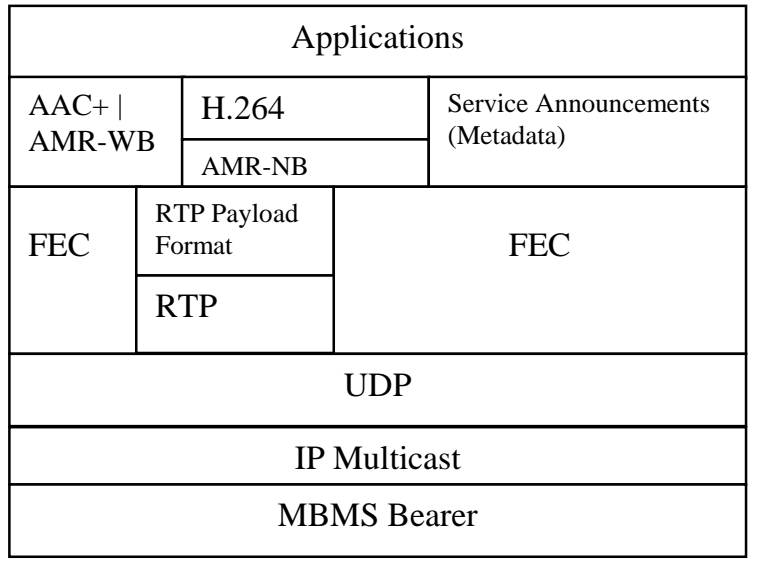

Figure 3: Software Architecture for MBMS streaming

\section{1) Streaming Delivery Protocols}

MBMS User Service Discovery/ Announcement are required for advertising MBMS Streaming in advance of, and potentially during, the User Service sessions. MBMS User Service Discovery/Announcement involves the delivery of fragments of metadata to many receivers in a suitable manner. The metadata itself describes details of services. A metadata fragment is a single uniquely identifiable block of metadata and could be described as an SDP file. The metadata consists of description of details about MBMS user services, MBMS user service sessions, associated delivery methods and service protection. The data types shall be specified using XML. The session description protocol (SDP) is also used to describe the multimedia delivery session.

The Real-time Transport Protocol (RTP) is used for delivery whereas RTCP is used for statistics on channel quality. The RTP and RTCP protocols are delivered over UDP/IP protocols. The MBMS bearer services are used to deliver over the MAC and physical layers.

\section{2) FEC model}

There is flexibility from the standard in terms of what FEC scheme to be used. If the selected FEC scheme does not fit, it can be modified. The generic mechanism for systematic FEC of RTP streams specifies two RTP payload formats, one for FEC source packets and one for FEC repair packets with their related signaling. After constructing the source block from the original RTP packets to be protected, the FEC encoder generates the desired amount of FEC protection data, i.e. encoding symbols. These encoding symbols are then sent using the FEC repair packet payload format to the receiver. The FEC repair packets use an SSRC different from the original RTP packets' SSRC, but are sent within the same RTP session. This would avoid non-continuous sequence numbering spaces for both the FEC repair packets and the original RTP packets.
The receivers recover the FEC source packets and places in a buffer for enough to receive the repair packets. The maximum time to store in the buffer is defined as the MIME type.

3) Media Codecs and Formats

For voice, AMR NB (Adaptive Multi-rate Narrow Band) Vocoder is supported. For Wideband voice, AMR WB is the mandatory codec for MBMS. The audio is supported by enhanced AAC+ (Advanced Audio Coding) and enhanced AMR-WB. The performance of AMR-WB is strong in low bit rate scenarios whereas $\mathrm{AAC}+$ is strong at high bit rate scenarios. The video codec that is recommended is H.264 (AVC) Baseline Profile Level 1b decoder however H.263 profile 0 level 45 decoder may also be used as it is supported by PSS.

\section{Proposed Scheme}

The proposed video coding scheme is designed to minimize dependencies, reduce error propagation, and perform receiver side adaptation such a frame dropping under low resource constraints.

\section{A. Detailed Scheme}

Figure 4 (a) shows the framing scheme for MPEG-4 SP video codec that has only I and P frames. In MPEG-4 sequences, I and P frames have to be decoded in the order they are received and skipping of even one frame would result in distortion that would propagate until an I frame is decoded. Figure 4 (b) shows the new scheme that we propose called periodic intra-frame based prediction (PIFBP), in which all the $\mathrm{P}$ frames in a group of pictures (GOP) use the same I-frame as a reference. With this structure, the $\mathrm{P}$ frames are dependent only on I frames and not on previous $\mathrm{P}$ frames. With this structure, the $\mathrm{P}$ frames can be decoded independent of other P Frames and the motion estimation and encoding is done based on the I Frame.

The proposed technique localizes errors in $\mathrm{P}$ frames to just those frames and eliminates distortion propagation. This improves error resilience but with a loss in quality. As the distance between $\mathrm{I}$ and $\mathrm{P}$ frame increases, the temporal correlation decreases thereby requiring more bits to accurately represent the image. If the target bit rate is fixed and sufficient bits are not available, this would result in loss of quality (PSNR).

Our experimental results indicate that the resulting drop in quality is not substantial and provides significant error resilience performance. This method can also be realized as a special case of $\mathrm{H} .264$ video coding (multi-frame prediction). 


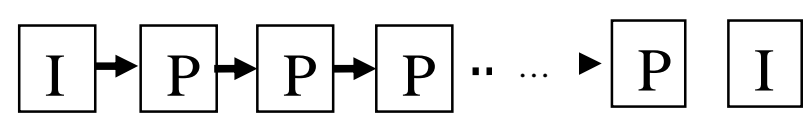

a) MPEG-4 Simple Profile

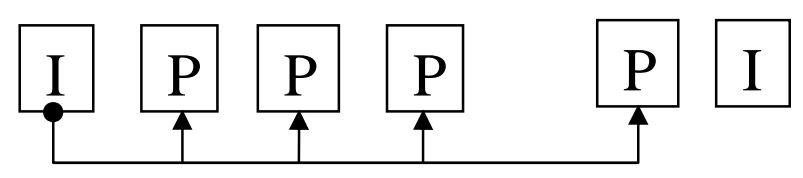

b) Periodic Intra Frame Based Prediction (PIFBP)

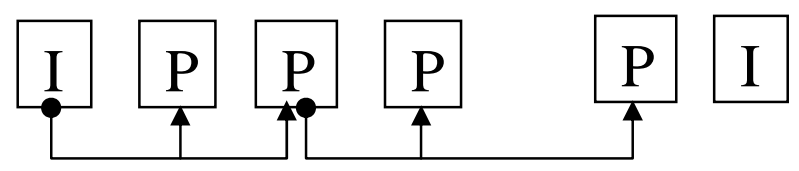

P) Periodic Anchor Frame Based Prediction
$($ PAFBP $)$

\section{Figure 4 Frame Structure of MPEG-4 (SP), PIFBP and} PAFBP schemes

Our experimental results indicate that the resulting drop in quality is not substantial and provides significant error resilience performance. This method can also be realized as a special case of H.264 video coding (multi-frame prediction).

Figure 4 (c) illustrates PAFBP scheme (Periodic anchor based frame prediction) that is an improvised variation of PIFBP scheme. In this scheme, I frame is encoded as an anchor frame at start of encoding. The anchor frames are periodically inserted once per anchor frame period and are predicted from previous anchor frames. The $\mathrm{P}$ frames within the anchor frame period are predicted from the previous anchor frame. If the Anchor Frame Period is small, there are high chances of $\mathrm{P}$ frames being temporally correlated to Anchor frames and would result in higher compression efficiency than I frames. For the foreman sequence, the PAFBP scheme improved PSNR by approximately $0.25-1 \mathrm{db}$ depending on the bit rate. With this approach, the anchor frames can be given priority over $\mathrm{P}$ frames thereby providing hierarchical structure.

\section{RESUlts AND Discussion}

\section{A. Experimental Setup}

In our experiments, the baseline software has been Motorola Labs Codec that has been used in several products and is compliant with the standards. The PIFBP and PAFBP schemes are implemented by modifying the baseline software. The Foreman and Carphone sequences at QCIF resolution are used for our experiments. The source video is at $30 \mathrm{fps}$ whereas the encoded video is at $15 \mathrm{fps}$ with target bit rate of 64,128 and $384 \mathrm{kbps}$. The use case for the experiments has been playback on a resource constrained device. The frame loss rate is varied from 1 to $25 \%$ and the results are obtained.

Figure 5 shows the plot of PSNR values of each individual frames of foreman sequence encoded using PIFBP and MPEG-4 SP profile codecs. The sequence is encoded at the rate of $128 \mathrm{kbps}$ and frame loss of $15 \%$ is simulated. With MPEG-4 baseline codec, the drop in quality represents the loss of a frame whereas PIFBP scheme maintains consistent quality despite frame loss. These results demonstrate that this scheme maintains the same quality under frame loss and performs really well. The test sequence used for simulations is FOREMAN sequence with QCIF resolution. The original sequence of 200 frames is captured at the rate of $30 \mathrm{fps}$ (frames per second) whereas the encoded frame rate is $15 \mathrm{fps}$. On the encoded bit stream, frame loss is simulated by dropping frames randomly using a random number generator. Such bit stream is decoded using MPEG-4 decoder and the resulting PSNR for 30 such iterations is averaged for accuracy purposes and is reported in this paper.

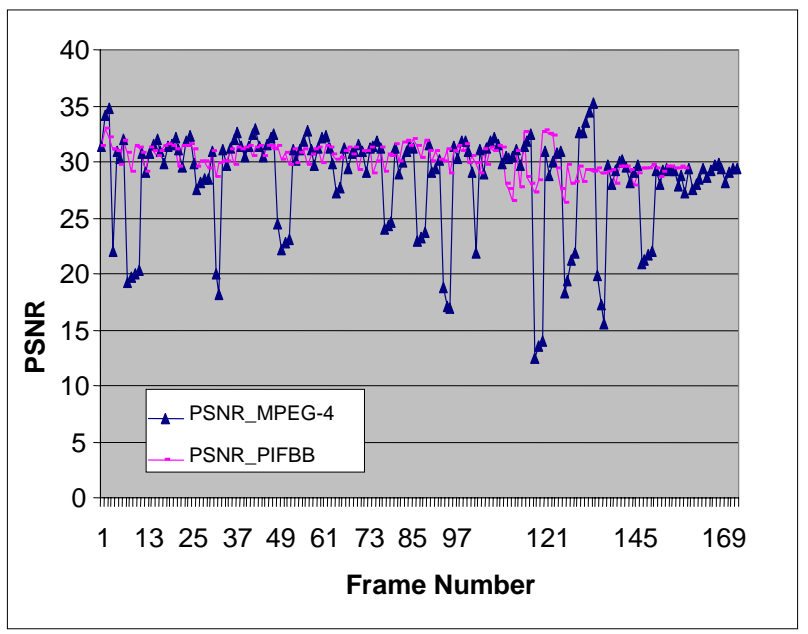

Figure 5: Comparison of PSNR figures for individual frame for MPEG-4 SP and proposed schemes for FOREMAN sequence at $128 \mathrm{kbps}, 15$ fps and $15 \%$ loss with VOP periodicity of 5 .

Figures 6-8 show the performance of PIFBP scheme compared with the MPEG-4 Simple Profile (SP) codec under frame loss conditions. The frame drop simulates two conditions: 1) frame drop because of bit errors and packet loss in the wireless network and 2) frame drop at the receiver due to limited battery or computing resources. Foreman sequence was used for the simulation and the frames are dropped randomly. By multiplying the loss rate and number of frames in the test sequences, the number of frames to be dropped is determined. A random number generator is used to figure out the frame that will be dropped. The experiments are repeated for bit rates of 64 kbps, $128 \mathrm{kbps}$ and $384 \mathrm{kbps}$ and for frame rates of 5, 10 
and 15. For simplicity, Error resilience features such as Intra Block refresh in MPEG-4 have been disabled. The periodicity of I frames is varied and the frame loss was simulated. For PIFBP, the period of I frames is chosen as 5.

Figure 6 shows the performance of PIFBP codec with Foreman sequence coded at $64 \mathrm{kbps}$ and $15 \mathrm{fps}$. This also shows that the new scheme is superior and maintains approximate same PSNR under loss conditions.

Shown in Figure 7 is the plot of PSNR values for individual frame coded at $128 \mathrm{kbps}$ with $15 \%$ frame loss for PIFBP scheme of VOP frequency of 5 and conventional scheme with VOP frequency of 5. Figure 7 depicts that there is less variation of PSNR in the new scheme as opposed to the Inter frame based prediction (MPEG-4 SP) coding and the average quality is much better in lossy conditions. It can also be deduced that during loss conditions of $5 \%$ or lower, the new scheme does worse than the old scheme however as the loss rate increases the resulting PSNR remains the same in the new scheme.

An adaptive scheme that uses both PIFBP and MPEG-4 SP would work better in all loss rate conditions. Depending on the channel conditions, if the loss rate is relatively low (less than $5 \%$ ), conventional scheme is used but is switched to the PIFBP scheme when loss rate is more than $5 \%$. The adaptive scheme depending on loss conditions would outperform both schemes in all conditions.

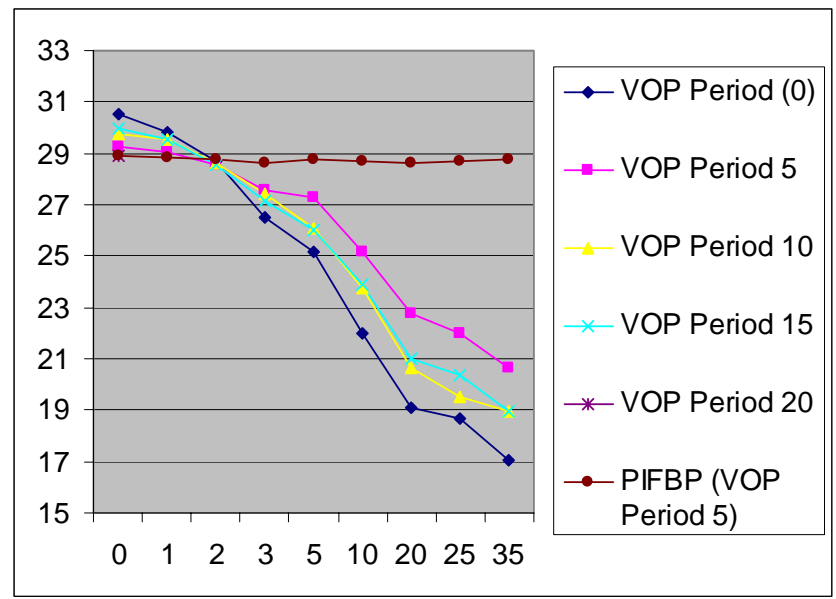

Figure 6: Performance under loss of MPEG-4 SP and proposed coding (Data rate $=64 \mathrm{kbps}, \mathrm{fps}=15$ )

Table 1 shows the performance comparison of PIFBP and MPEG-4 simple profile coding. The results are shown for no frame loss and $20 \%$ frame loss conditions. A use case of $20 \%$ frame loss is chosen to illustrate the scenario of a mobile device that can support video playback of $12 \mathrm{fps}$ whereas the content is encoded at $15 \mathrm{fps}$ and that constitutes $20 \%$ loss.

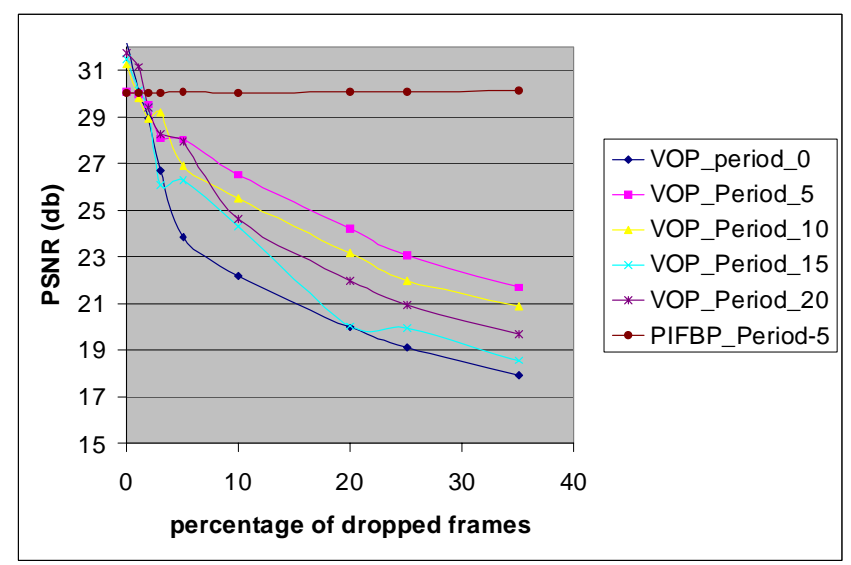

Figure 7 Performance under loss of MPEG-4 SP and proposed coding (Data rate $=128 \mathrm{kbps}, \mathrm{fps}=15 \mathrm{fps}$ )

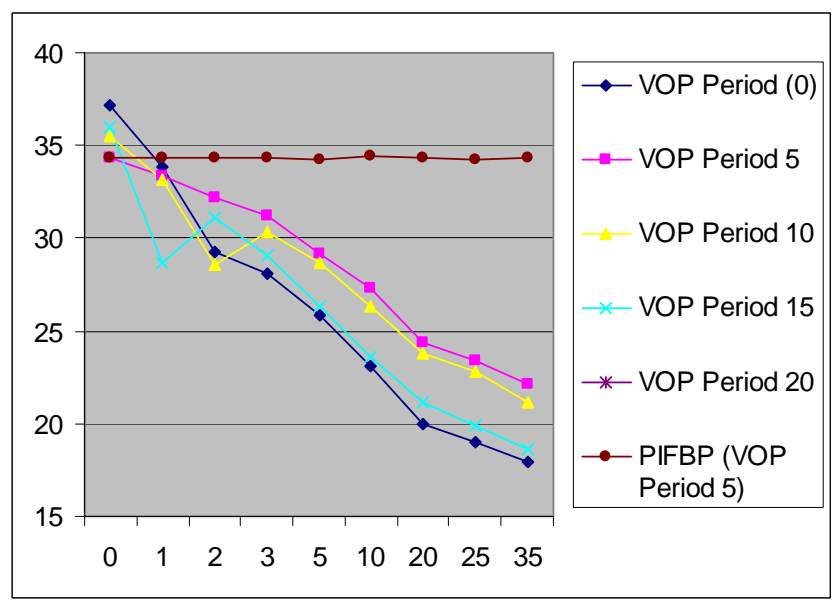

Figure 8 Performance under loss of MPEG-4 SP and proposed coding (Data rate $=384 \mathrm{kbps}, \mathrm{fps}=15$ )

This table shows that the proposed prediction is less efficient than MPEG-4 SP prediction; however, in lossy conditions, PIFBP performs much better and gives consistent quality. This is due to the error resilient framing structure of PIFBP and hides the device conditions from the presentation. To simulate losses in constrained devices, that do not have processing power to render high frame rate video bit stream, a frame loss simulation are conducted. The frame loss simulation is considered during scenarios such as devices with lower processing power, congestion during transport, the frames are either lost or skipped during playback. The frames are also skipped to achieve synchronization with the audio. The skipping of $\mathrm{P}$ frame or I frame in the conventional schemes would cause distortion and this would propagate until the decoding of I frame. The skipping of B Frames does not cause any distortion however they are not considered due to the complexity involved and are not required for Simple Profile

In Periodic Anchor Frame Based Prediction (PAFBP) the overall efficiency could be improved over PIFBP by having anchor frames that depend on previous anchor frames [11]. This would result in separate threads, one is thread of 
anchor frames that is less frequent and is preserved using the unequal error protection or retransmissions. Another thread is frames that are between two anchor frames and are dependent on anchor frames.

Table 1: Effect of periodicity and frame losses on PIFBP, PAFBP and MPEG-4 SP for Foreman sequence (128 kbps) at $15 \mathrm{fps}$

\begin{tabular}{|c|c|c|c|c|c|c|}
\hline $\begin{array}{l}\text { VOP } \\
\text { Period } \\
\text { icity }\end{array}$ & $\begin{array}{l}\text { PSNR } \\
\text { (MPEG- } \\
4 \text { SP) } \\
\text { (Error } \\
\text { Free) } \\
\end{array}$ & $\begin{array}{l}\text { PSNR } \\
\text { (PIFBP } \\
\text { ) } \\
\text { (Error } \\
\text { Free) } \\
\end{array}$ & $\begin{array}{l}\text { PSNR } \\
\text { (PAFB } \\
\text { P) } \\
\text { (Error } \\
\text { Free) } \\
\end{array}$ & $\begin{array}{l}\text { PSNR } \\
\text { (MPEG- } \\
4 \text { SP) } \\
(20 \% \\
\text { loss }) \\
\end{array}$ & $\begin{array}{l}\text { PSNR } \\
\text { (PIFBP } \\
) \\
(20 \% \\
\text { loss) } \\
\end{array}$ & $\begin{array}{l}\text { PSNR } \\
\text { (PAFBP) } \\
(20 \% \\
\text { loss) }\end{array}$ \\
\hline 5 & 30.51 & 30.05 & 30.05 & 25.42 & 30.05 & 30.05 \\
\hline 10 & 31.25 & 29.88 & 30.25 & 24.04 & 29.87 & 30.25 \\
\hline 15 & 31.52 & 29.65 & 30.27 & 22.62 & 29.65 & 30.27 \\
\hline 20 & 31.73 & 29.21 & 30.34 & 22.85 & 29.21 & 30.3 \\
\hline 0 & 32.19 & 27.85 & 30.32 & 20.81 & 27.85 & 30.28 \\
\hline
\end{tabular}

For the foreman sequence coded at $128 \mathrm{kbps}$, the PAFBP and PIFBP schemes result in same error free quality for VOP period of 5. Note that for PIFBP scheme, an I Frame is repeated every VOP period whereas for PAFBP, the Anchor frame is repeated every VOP period. When the VOP period increases, the PAFBP scheme maintains the quality whereas the PIFBP scheme loses in quality.

For higher bit rates, the PAFBP scheme outperforms PIFBP as shown in the following Figure. Car phone and Foreman sequences were used for this experiment where VOP period is varied for $64 \mathrm{kbps}, 128 \mathrm{kbps}$ and $384 \mathrm{kbps}$ bit rates. Under loss conditions, the PAFBP would maintain consistent quality (overall PSNR) as PIFBP scheme so we do not wish to repeat the results.

Figure 9 shows the performance of PAFBP, PIFBP and MPEG-4 SP baseline schemes for Carphone sequence encoded at the rate of 64 and $384 \mathrm{kbps}$. In this sequence, for PAFBP scheme, the Anchor Frame period is kept at 5 and it improves the performance over PIFBP scheme. Figure 10 shows the performance for Foreman sequence coded at 64 and 384 kbps.

Even though, the PAFBP and PIFBP perform similarly for VOP period of 5, as VOP period increases and Anchor Frame period is kept constant at 5, the coding performance improves for PAFBP. It performs similar to MPEG-4 Baseline. However, there is a coding hit compared to MPEG-4 baseline and results in an error resilient structure.

The proposed schemes can be applied video reception over networks during lossy conditions and also to end devices have limited processing power and capability. In networks with lossy conditions, burst errors could result in dropping of a frame. Also, due to VLC, a bit error could result in losing synchronization and dropping of the frames. In such case, in the new scheme, the frame could be skipped or simple error concealment technique could be applied without impact on overall quality. This is not noticeable by the end user as opposed to distortion propagation that is noticeable as it spans several frames.

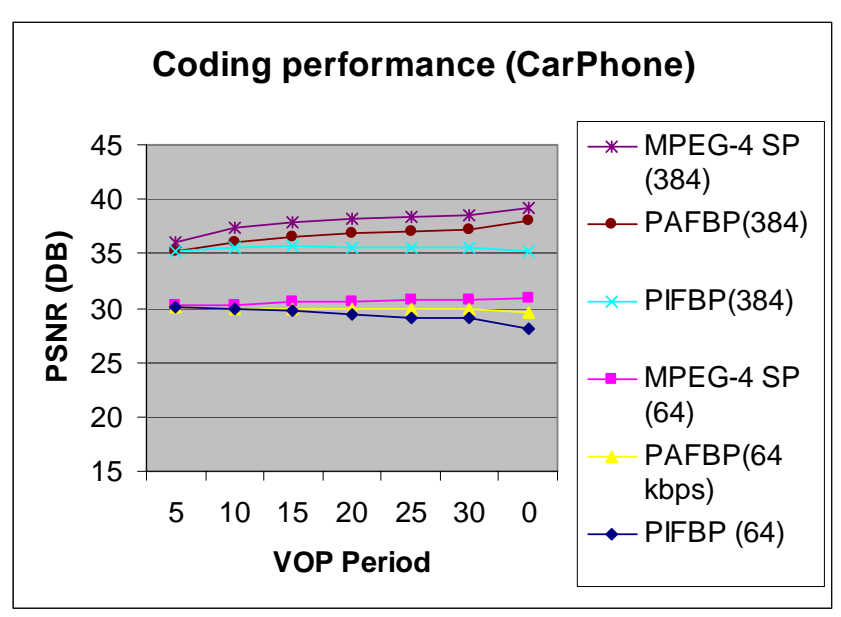

Figure 9 Coding Performance for Carphone

We compared the proposed method with a commonly used error resilience technique of refreshing intra blocks. The MPEG-4 SP codec using intra Block Refresh (IBR) is compared with the proposed approaches. The use of IBR in MPEG-4 SP improves quality in frame loss conditions [7] as shown in

Table 2 but overall technique is still inferior to the PIFBP or PAFBP scheme.

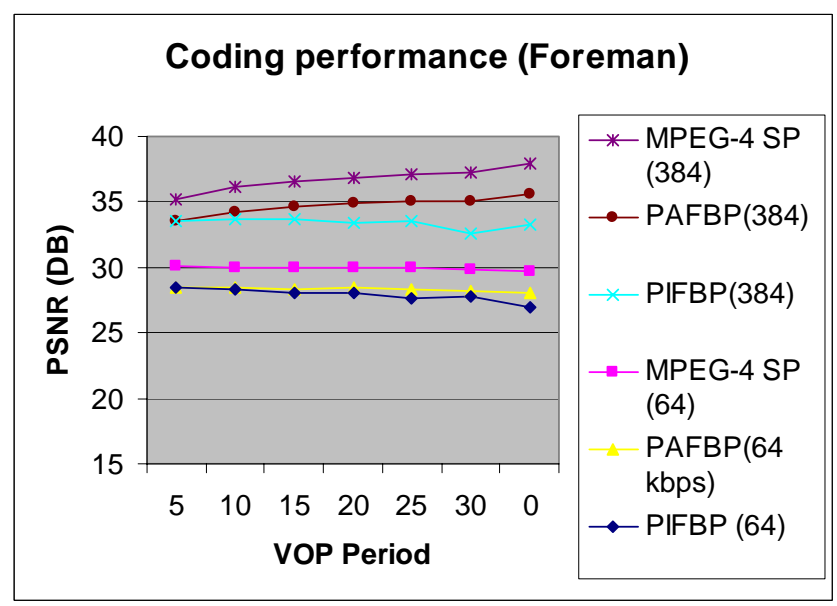

Figure 10 Coding Performance for Foreman 
Table 2: Effect of Intra Macro Block Refresh rates on different frame loss rates of Inter Frame prediction (Foreman, $128 \mathrm{kbps}, 15 \mathrm{fps}$ ) (random Losses averaging over 30 tries)

\begin{tabular}{|l|l|l|l|l|}
\hline $\begin{array}{l}\text { Rando } \\
\mathrm{m} \\
\begin{array}{l}\text { Loss } \\
\text { Rate }\end{array}\end{array}$ & $\begin{array}{l}\text { PSNR(0 } \\
\text { ) }\end{array}$ & $\begin{array}{l}\text { PSNR( } \\
\text { 0) with } \\
\text { Intra } \\
\text { Refresh }\end{array}$ & $\begin{array}{l}\text { PSNR(0) } \\
\text { with Intra } \\
\text { Refresh }+ \\
\text { RVLC + } \\
\text { Data } \\
\text { Partitioning }\end{array}$ & $\begin{array}{l}\text { PIFBP } \\
\text { Scheme } \\
\text { with no } \\
\text { error } \\
\text { resilience } \\
\text { features }\end{array}$ \\
\hline 0 & 32.19 & 31.99 & 31.99 & 30.05 \\
\hline 1 & 30.08 & 30.52 & 30.54 & 29.92 \\
\hline 2 & 28.92 & 29.73 & 29.12 & 29.85 \\
\hline 3 & 26.70 & 28.50 & 28.76 & 29.73 \\
\hline 5 & 23.84 & 26.98 & 27.04 & 29.39 \\
\hline 10 & 22.18 & 24.25 & 24.34 & 28.73 \\
\hline 20 & 20 & 21.49 & 21.63 & 27.60 \\
\hline 25 & 19.12 & 20.89 & 20.52 & 27.59 \\
\hline 35 & 17.91 & 19.57 & 19.69 & 26.56 \\
\hline
\end{tabular}

As shown in table 2, adding the feature of random Intra block refresh lowers PSNR of $0.2 \mathrm{db}$ for Error free quality but improves overall quality (up to $3 \mathrm{db}$ at $5 \%$ loss) when there is loss. Since frame loss is simulated, the other MPEG-4 Error resilient features such as RVLC, Data partitioning [8] do not add any significant improvement in quality during loss. The PIFBP Scheme performs much better in frame loss scenarios.

\section{B. Discussion}

The proposed scheme does offer much better performance than conventional scheme in frame loss conditions. It offers consistent performance in error-free and error environments by shielding the user from device limitations. The proposed scheme is quite simple and in case of errors in a $\mathrm{P}$ frame, the frame could be dropped without impacting the rest of the Video. The existing error resilient techniques require processing power and would drain the current whereas the new scheme consumes less power. The resulting gains due to error concealment are depend on where error has occurred and are hard to quantify. The dependency makes it more difficult and can be avoided with PIFBP/PAFBP.

This frame skipping can be done at any point in network such as server, intermediate points and client. The RTP/UDP/IP scheme can be used to packetize one frame per packet and can be dropped at server for reasons such as server load, congestion on the network and bandwidth adaptation. Since the packets are on frame boundaries, they could be dropped at intermediate points because of network congestion etc. The client can also drop frames because of power, bandwidth and other constraints. The new scheme does much better over traditional schemes during such losses and is scalable. The PIFBP scheme is useful when the capabilities of the end devices are not known. If the content is placed on the server with a target frame rate with PIFBP, devices that can support lower and higher frame rates can download the same content and the content can adapt to the device characteristics. Also, if there are devices that belong to the same network but have different processing capabilities, interoperability is an issue. With the new scheme, the content created once can be used for devices with diverse capabilities.

In the PIFBP/PAFBP, based on the channel conditions the distance between the anchor frames could be reduced thereby improving the overall performance. With MBMS applications, multicasting creates an environment where receivers have minimal feedback to the sender and the sender has almost no ability to respond to individual receiver feedback. In such environments, a video coding scheme with reduced dependencies will allow error localization and reduces reliance on the sender for corrective measures. The multicast applications can further improve the reliability by partitioning the Intra or anchor frames in video bitstream into a separate multicast group with stronger FEC. Creating a separate multicast group for Intra/anchor frames also enables receivers to quickly join a multicast session.

\section{Conclusion}

In this paper, we propose a video codec that addresses the two key issues in video delivery to mobile devices: 1) error resilience and 2) resource adaptive playback. We show that the proposed codec is simple to implement on low-resource terminals such as mobile phones and outperforms MPEG-4 SP coding under lossy conditions. Since the proposed scheme can be adapted for any hybrid video codec, no new IP is necessary. The proposed codec can be implemented at an application level even on mobile devices that have been deployed. We also show that the proposed coding scheme is more error resilient than MPEG-4 SP with Intra Block Refresh technique. The minimization of dependencies due to the proposed approach improves the quality of video over MBMS and allows receiver side adaptation.

\section{REFERENCES}

[1] Yao Wang and Qin-Fan Zhu, "Error Control and Concealment for Video Communications: A Review", Proceedings of the IEEE, Vol. 86, NO. 5, May. 1998.

[2] Bansal, Pankaj, Narendran, M. R. and Manohar Murali N. K., "Im-proved Error Detection and Localization techniques for MPEG-4 Video", International 
Conference on Image Processing., Vol. 2, Pages:II-693 - II-696, 22-25 Sept. 2002

[3] Chun g-How, J.T.H. and Bull, D.R., "Robust H.263+ video for Real-Time Internet Applications", Proceedings of International Conference on Image Processing, vol. 3, pp. 544-547, Sept. 2000.

[4] S. Wenger, G. Knorr, J. Ott, and F. Kossentini, "Error resilience support in H.263+," IEEE Trans. Circuits Syst. Video Technol., vol. 8, no. 6, pp. 867-877, Nov. 1998.

[5] Wiegand, T., Sullivan, G.J., Bjntegaard, G. and Luthra, A., "Overview of the H.264/AVC video coding standard", IEEE Transactions on Circuits and Systems for Video Technology, Volume: 13 , Issue: 7 , July 2003, pp. $560-576$

[6] Freytes, M., Rodriguez, C.E., Marques, C.A., "Realtime H.263+ video transmission on 802.11 wireless LANs", Proceedings. International Conference on Information Technology: Coding and Computing, pp. 125-129, 2-4 April 2001.

[7] Zhao, C, Ngan, K.N, Zhang, J, Mathew, R.and Zhang, $\mathrm{X}$., "Using inter-frame dependence history to select intra-refresh blocks" Electronics Letters, Volume: 38 , Issue: $22-24$, Oct 20.

[8] Talluri. R, "Error Resilient coding in the ISO MPEG-4 standard", IEEE Communications Magazine, Vol. 36, Issue. 6, pages 112-119, June 1998.

[9] Brailean James, "Wireless Multimedia utilizing MPEG-4 Error resilient Tools", IEEE Transactions, 1999.

[10] Pereira Fernando, "MPEG-4 Video Subjective Test Procedures and Results", IEE Transactions on Circuits and Systems for Video Tech-nology", Vol.7, No. 1, February 1999.

[11]P. Sanigepalli, H. Kalva, and B. Furht, "An Improved Video Codec Supporting Error Resilience and Resource Adaptation," Proceedings of the IEEE International Conference on Consumer Electronics, January 2005, pp. 311-312.

[12]P. Sanigepalli, H. Kalva, and B. Furht, "Application of Video Error Resilience Techniques for Mobile Broadcast Multicast Services (MBMS)," Proceedings of the 6th IEEE International Symposium on Multimedia Software Engineering, December 2004, pp. 507-512.

[13]3GPP TS 23.246: "Multimedia Broadcast/Multicast Service (MBMS); Architecture and functional description".

[14]3GPP TS 26.346 "Multimedia Broadcast/Multicast Service (MBMS); Protocols and codecs". 